beauticians, cleaners, a millwright, a waitress and several workers that either decanted or mixed the chemicals.

Discussion $\mathrm{MCI} / \mathrm{MI}$ and $\mathrm{MI}$ are important occupational allergens and should be tested using the correct concentration to avoid misdiagnosing cases. The importance of keeping abreast with what is happening in the field was highlighted by the changes implemented which improved the detection of these allergens.

\section{7b FROM WORKPLACE TO HOME ENVIRONMENT: SPREADING OF MOUSE ALLERGENS BY LABORATORY ANIMAL WORKERS}

H Kube, L Wengenroth*, J Gerlich, G Dietrich-Gümperlein, R Schierl, D Nowak, K Radon. Institute and Outpatient Clinic for Occupational, Social and Environmental Medicine, Munich, Germany

\subsection{6/oemed-2018-ICOHabstracts. 144}

Introduction Laboratory animal workers (LAW) working with mice are exposed to mouse allergens (MA). Exposure to MA can lead to occupational allergies and asthma. If MA are spread to home environments, the longer duration of exposure might increase the risk for allergic symptoms. Little is known about the spreading of MA. This study aimed to assess:

- whether spreading of MA from workplace to home environment takes place,

- which factors increase spreading of MA.

Methods In a cross-sectional study we took dust samples from the homes of 107 LAW and 13 controls. From 90 LAW we took additional dust samples from their working place. Samples were analysed using mus $m 1$ ELISA kits. Through a questionnaire we assessed socio-demographic data, allergies and cleaning habits. In LAW we also assessed types of cages used, work tasks and protective clothing.

Results MA concentration was higher in home environments of LAW (median (ng mus $\mathrm{m} 1$ )=11.3) than in controls (median $=1.1 ; \mathrm{p}=0.016 ;$ Kruskal-Wallis test). The highest workplace MA concentration was found in the scullery (median $=145,0000.0$ ), followed by the changing rooms (median $=10.2)$ and staffrooms (median $=7.5$ ). MA concentration was higher in homes of LAW who fulfilled cleaning tasks (cleaning of cages, floors, etc.) $(p=0.034)$ and who changed their linen at home less than once a month $(p=0.024)$. MA concentration at home was not associated with duration of mouse contact $(\mathrm{p}=0.909)$ and age of sleeping mattress at home $(p=0.649)$.

Discussion Spreading of MA from workplace to home environment takes place. LAWs who fulfilled cleaning tasks were found to have higher MA concentration at home. Special focus should be given to reduce MA concentration during cleaning in laboratory animal facilities.

\section{C INHALED FINE PARTICLES INDUCE ALLERGIC INFLAMMATION IN THE LUNGS}

\footnotetext{
1,2,3 E Kuroda*, 1,2 KJ Ishii. 'Laboratory of Adjuvant Innovation, Centre for Vaccine and Adjuvant Research (CVAR), National Institutes of Biomedical Innovation, Health and Nutrition, Osaka, Japan; ' 2 Laboratory of Vaccine Science, WPI Immunology Frontier Research Centre (IFReC), Osaka University, Osaka, Japan; ${ }^{3}$ Japan Science and Technology Agency (JST), PRESTO, Japan
}

10.1136/oemed-2018-ICOHabstracts. 145
It is known that inhaled fine particles such as particle pollutions are associated with allergic responses. Many reports have shown that some particulates function as adjuvant to enhance immune responses, however its underlying mechanisms are still unclear. Here, we will show how particulates induce immune responses in the lungs.

We used aluminium salt (alum) and crystalline silica as inflammatory particulate. These inflammatory particulates stimulated alveolar macrophage to release interleukin-1 $\alpha$ (IL$1 \alpha$ ) through cell death. We also observed IL- $1 \alpha$ release in the bronchoalveolar lavage fluid (BALF) after intratracheal instillation (i.t.) of inflammatory particulate alum in vivo. Released IL- $1 \alpha$ in the lungs was thought to be a damage-associated molecular pattern (DAMP) and contributed to antigen-specific IgE production in mouse model of particulate-induced allergic inflammation. In addition, we found the lymphoid cluster formations in the lungs after i.t. alum instillation and allergen exposure. This lymphoid cluster was considered to be inducible bronchus-associated lymphoid tissue (iBALT), and we suggest that iBALT formation might be an important role for local IgE responses in the lungs.

These results indicate that inhalation of particulates and allergen exposure induces and exacerbate allergic responses though the unique immune responses in the lungs such as alveolar macrophage death and iBALT formation.

\section{7d BEEKEEPER ALLERGY RISK AND WORK SUITABILITY}

M Di Gioacchino*, M Di Giampaolo, C Petrarca. Unit of Immunotoxicology and Allergy, CSIMET, G. d'Annunzio University, Chieti, Italy

\subsection{6/oemed-2018-ICOHabstracts.146}

Introduction The risk for a beekeeper to become allergic to bee venom is higher than the general population. The possibility that they develop an allergy is greater when rarely bite with $2 / 3$ month interval. On the contrary, when bites are simultaneous or tight over time the probability of allergy decreases. Beekeepers who receive more than 200 stings do not develop venom allergy. In general, subjects developing a reaction limited to the skin have $70 \%$ possibility of a spontaneous recovery. On the contrary, people haveing a systemic reaction tends to show the same or greater reaction when restung.

Discussion Work suitability of bee allergic beekeepers, which had a systemic reaction is a relevant problem for occupational doctors. They should be prescribed appropriate DPI and provided of auto-injectable adrenaline as emergency treatment, sure that they received the necessary training. Furthermore, it is desirable that every allergic subject receives a desensitising treatment, as it is efficacious in $95 \%$ of patients. The treatment will last for 5 years, except for subjects with high basal tryptase who should be treated for a life-time, as the risk of anaphylaxis reappears after the discontinuation of an already effective immunotherapy. The cardiac involvement during anaphylaxis is very frequent with the picture of Kounis Syndrome (KS), characterised by infarction induced by the effects on coronaries of mast cell chemical mediators. In particular, histamine, usually vasodilator agent, can induce vasoconstriction subjects with coronary endothelial lesions. In this situation, the coronary spasm worsens the perfusion of a tachy heart, already with increased blood demand, with a potentially lifethreatening ischemia. Furthermore, in a subject developing a 